\title{
Microcarcinoma papilar de tiroides: ¿es adecuada la selección para protocolo de vigilancia activa?
}

\author{
Papillary thyroid microcarcinoma: is selection suitable for active \\ surveillance protocol?
}

\author{
David Mauricio Figueroa-Bohórquez 1 (D), Pilar Carola Pinillos-Navarro² (D), \\ Jorge Alberto Martínez-Martínez ${ }^{\mathbb{D}} \mathbb{D}$, Daniela Casallas-Cristancho ${ }^{3} \mathbb{D}$, \\ Jenniffer Juliana León-Acero ${ }^{3} \mathbb{D}$, Diego Alejandro Ardila-Torres ${ }^{4} \mathbb{D}$, Giancarlo Buitrago ${ }^{5} \mathbb{D}$, \\ Sergio Fabián Zuñiga-Pavia ${ }^{6}$ (D) \\ 1 Médico, residente de Cirugía general, Universidad Nacional de Colombia, Bogotá, D.C., Colombia \\ 2 Médica, especialista en Cirugía general, Universidad Nacional de Colombia, Bogotá, D.C., Colombia \\ 3 Médico, Universidad Nacional de Colombia, Bogotá, D.C., Colombia \\ 4 Médico, especialista en Cirugía general y Cirugía de Cabeza y cuello, Hospital Universitario Nacional de Colombia, Bogotá, D.C., Colombia \\ 5 Médico, magíster en Epidemiología Clínica y Economía, Universidad Nacional de Colombia, Bogotá, D.C., Colombia \\ 6 Médico, especialista en Cirugía general y Cirugía de cabeza y cuello; jefe, departamento de Cirugía de Cabeza y Cuello, Hospital \\ Universitario Nacional de Colombia, Bogotá, D.C., Colombia
}

Trabajo ganador del tercer puesto en el Concurso de Investigación en Cirugía "José Félix Patiño Restrepo" del Congreso Semana Quirúrgica Nacional 2020 de la Asociación Colombiana de Cirugía. Bogotá, D.C, Colombia. Noviembre de 2020.

\section{Resumen}

Introducción. Los microcarcinomas papilares de tiroides son tumores de hasta 10 mm en su diámetro mayor. Su tratamiento es sujeto de debate y se propone, desde seguimiento clínico, hasta intervención quirúrgica temprana. Este estudio buscó identificar factores de riesgo relacionados con compromiso ganglionar, que permitan una mejor selección de los pacientes en nuestro medio, en quienes se propone manejo quirúrgico inmediato o vigilancia activa, en consonancia con la clasificación del riesgo de progresión.

Métodos. Estudio de cohorte analítica ambispectiva que incluyó pacientes con microcarcinoma papilar de tiroides llevados a tiroidectomía más vaciamiento central. Se caracterizó la población y se realizó un análisis de regresión logística multivariado para definir factores preoperatorios asociados al compromiso ganglionar. Adicionalmente, se evaluó de manera retrospectiva la eventual asignación a grupos de riesgo de progresión, según los criterios de Miyauchi, y su comportamiento respecto al estado nodal.

Resultados. Se incluyeron 286 pacientes. El 48,9 \% presentó compromiso ganglionar, y de estos, el 33,5 \% presentó compromiso ganglionar significativo, que modificó su clasificación de riesgo de recaída. De estos

Fecha de recibido: 15/09/2020 - Fecha de aceptación: 15/11/2020 - Fecha de publicación en línea: 12/02/2021

Correspondencia: David Mauricio Figueroa Bohórquez, Calle 61 \# 3D- 15, Bogotá, D.C., Colombia. Teléfono: 3153741070

Correo electrónico: damfigueroabo@unal.edu.co

Citar como: Figueroa-Bohórquez DM, Pinillos-Navarro PC, Martínez-Martínez JA, Casallas-Cristancho D, León-Acero JJ, ArdilaTorres DA, et al. Microcarcinoma papilar de tiroides: ¿es adecuada la selección para protocolo de vigilancia activa? Rev Colomb Cir. 2021;36:248-56. https://doi.org/10.30944/20117582.838

Este es un artículo de acceso abierto bajo una Licencia Creative Commons - BY-NC-ND https://creativecommons.org/licenses/by-ncnd/4.0/deed.es 
últimos, el 59,5 \% hubiesen sido manejados con vigilancia activa, según los criterios propuestos por Miyauchi. Se identificó que la edad menor de 55 años, los ganglios sospechosos en la ecografía y los nódulos mayores de $5 \mathrm{~mm}$, se relacionan con compromiso ganglionar significativo.

Discusión. El manejo quirúrgico inmediato parece ser una opción adecuada para pacientes con sospecha de compromiso ganglionar en ecografía preoperatoria, pacientes menores de 55 años y nódulos mayores de $5 \mathrm{~mm}$. Es posible que los actuales criterios para definir vigilancia activa no seleccionen adecuadamente a los pacientes en nuestro medio.

Palabras clave: neoplasias de la tiroides; cáncer papilar tiroideo; carcinoma papilar; metástasis linfática; tiroidectomía; disección del cuello.

\begin{abstract}
Introduction. Papillary thyroid microcarcinomas are tumors up to $10 \mathrm{~mm}$ in greatest diameter. Its treatment is subject of debate, and it is proposed from clinical follow-up to early surgical intervention. The aim of the study was to identify risk factors related to lymph node involvement, which allow a better selection of patients in our setting, in whom immediate surgical management or active surveillance is proposed, in accordance with the classification of risk of progression.
\end{abstract}

Methods. Ambispective analytic cohort study that included patients with papillary thyroid microcarcinoma who underwent thyroidectomy and central dissection. The population was characterized and a multivariate logistic regression analysis was performed to define preoperative factors associated with lymph node involvement. Additionally, eventual assignment to progression risk groups, according to the Miyauchi criteria, and their nodal state were evaluated.

Results. 286 patients with papillary thyroid microcarcinoma were included. Among them, $48.9 \%$ had lymph node disease, and $33.5 \%$ had a significant lymph node disease that increased their relapse risk classification. Of the latter, $59.5 \%$ could have had a conservative treatment, under Miyauchi's criteria. For ages $<55$ years old, suspect nodes in ultrasound and nodules $>5 \mathrm{~mm}$ were identified as related to significant lymph node involvement.

Discussion. Immediate surgical management appears to be an appropriate option for patients with suspected lymph node involvement on preoperative ultrasound, patients younger than 55 years and nodules larger than $5 \mathrm{~mm}$. It is possible that the current criteria for defining active surveillance do not adequately select patients in our setting.

Keywords: thyroid neoplasms; papillary thyroid cancer; papillary carcinoma; lymphatic metastasis; thyroidectomy; neck dissection.

\section{Introducción}

El término microcarcinoma papilar de tiroides (MCPT) hace referencia a los tumores con tamaño de hasta $10 \mathrm{~mm}$ en su diámetro mayor, los cuales representan más del $50 \%$ de todos los casos de cáncer de tiroides ${ }^{1-4}$. Tiene un curso aparentemente benigno $y$, a pesar de su alta prevalencia, la mortalidad específica de este tipo de tumores es menor del $1 \%$, la recurrencia locorregional es de 2 a $6 \%$ y la recurrencia a distancia se presenta entre el 1 y el $2 \%$ de los pacientes ${ }^{5,6}$. En Colombia encontramos un único reporte de metástasis a distancia en un paciente masculino de 49 años, quien se presentó con metástasis a fémur ${ }^{7}$.

En la actualidad, el manejo de los MCPT no está totalmente establecido, por lo cual está sujeto a debate donde se incluye una amplia variedad de conductas, que van desde la estrategia de vigilancia activa hasta el tratamiento quirúrgico temprano. Alrededor de la opción quirúrgica, se discute la extensión óptima de la misma y el papel de vaciamiento ganglionar electivo de los niveles VI y VII ${ }^{6,8}$. 
Se ha demostrado que los MCPT suelen tener un curso indolente en la mayoría de los casos ${ }^{8}$. En un seguimiento clínico de 340 pacientes con MCPT, se encontró que solamente el 6,4 y 15,9 \% de los pacientes presentaron crecimiento tumoral de $3 \mathrm{~mm}$ o más, a los 5 y 10 años respectivamente. En el mismo estudio se identificó compromiso nodal en el 1,4 y 3,4\% de los pacientes, a los 5 y 10 años respectivamente. Con base en estos resultados, se propone no realizar manejo quirúrgico inmediato e iniciar seguimiento clínico estricto a aquellos pacientes con MCPT que no tengan características de alto riesgo, las cuales son: extensión extracapsular en la ecografía, compromiso ganglionar clínico o imagenológico, citología con hallazgos de agresividad, o crecimiento del nódulo en seguimiento ${ }^{9}$. La carencia de estas características ha sido denominada por múltiples autores como microtumor papilar de tiroides, para definir un tumor cuyo comportamiento es de un curso más predecible ${ }^{10}$.

En Colombia, se informó el estudio de una cohorte prospectiva de 102 pacientes, en el que se evalúo la experiencia de la vigilancia activa en carcinoma tiroideo de bajo riesgo, incluyendo a los pacientes con tumores encapsulados, menores de $1,5 \mathrm{~cm}$ y sin evidencia de compromiso nodal, sin tener en cuenta género, ni edad ${ }^{11}$. La media de seguimiento fue de 13,9 meses y el 32,3\% de los pacientes tuvieron un seguimiento mayor a 24 meses. El 10,8 \% de los pacientes tuvieron un crecimiento tumoral mayor a $3 \mathrm{~mm}$, el $14,7 \%$ un crecimiento mayor del $30 \%$ en su diámetro mayor, y el 25,5 \% presentó un crecimiento mayor que el $50 \%$ de su volumen durante la vigilancia activa. Estos datos están en rangos similares a los de otros estudios internacionales y sugieren la posibilidad de realizar seguimiento clínico a los pacientes con MCPT, de forma segura, en nuestro medio ${ }^{12,13}$.

Un estudio descriptivo realizado en Bogotá, Colombia, que reunió 509 pacientes con cáncer de tiroides, concluyó que la falta de una clasificación preoperatoria para establecer el riesgo de recaída o de mortalidad, la presencia de una variable histológica agresiva y las deficiencias del sistema de salud, no permiten un adecuado seguimiento de los pacientes con patología tumoral tiroidea, por lo cual, pueden ser justificación para dar manejo quirúrgico agresivo como tratamiento inicial ${ }^{14}$.

Cuando se opta por el tratamiento quirúrgico temprano, se debe definir la extensión de la resección tiroidea y la necesidad de vaciamiento ganglionar central. El compromiso ganglionar regional de los MCPT oscila entre el 14 y el $46 \%$, por lo que se debate el rol del vaciamiento central sistemático ${ }^{15-17}$. Tanto las guías ATA 2015, como otros autores, recomiendan realizar un vaciamiento ganglionar central solamente en tumores T3 y T4 ${ }^{18,19}$, no obstante, algunos autores afirman que realizar vaciamiento ganglionar central sistemático en tumores T1 y T2 puede reducir la recurrencia locorregional del 4,5 - 8,6 \% hasta el $2,5-5,9 \%{ }^{20}$.

El objetivo de este estudio fue identificar los factores de riesgo clínicos o paraclínicos preoperatorios relacionados con compromiso ganglionar central general y compromiso ganglionar central significativo para nuestra población, así como analizar el compromiso nodal, en función de la asignación retrospectiva de los pacientes a grupos de riesgo de progresión, según los criterios propuestos por Miyauchi ${ }^{20}$.

\section{Métodos}

Estudio de cohorte ambispectivo de pacientes con patología tiroidea, llevados a cirugía por el servicio de cirugía de cabeza y cuello, en el Hospital Universitario Nacional de Colombia, en la ciudad de Bogotá, D.C., Colombia, desde enero de 2017 hasta julio de 2020. Para fines prácticos, en este estudio se utilizó el término MCPT de bajo riesgo, como ha sido definido a nivel mundial.

Se incluyeron todos los pacientes mayores de 18 años de edad, con diagnóstico de carcinoma papilar de tiroides, con tumores de hasta $10 \mathrm{~mm}$ de acuerdo con el reporte de la anatomía patológica, llevados a tiroidectomía total y vaciamiento ganglionar central, intervenidos por primera vez en la institución y que hubiesen rechazado la propuesta de vigilancia activa en caso de ser candidatos a este manejo. Se excluyeron los pacientes en quienes se identificó al ingreso metástasis a 
distancia o compromiso ganglionar lateral y los pacientes con disentimiento informado para el uso de datos en la fase prospectiva de la recolección de los mismos, que inició en febrero del 2020. Se recolectó la información a través de un instrumento donde se incluyeron las variables de interés en momentos secuenciales de la atención al paciente, desde la consulta prequirúrgica, incluyendo cirugía y reporte de patología, hasta los controles posoperatorios.

Se realizó un análisis descriptivo de las características de la población con medidas de frecuencia y de tendencia central, las cuales incluyeron variables sociodemográficas, clínicas y desenlaces. Adicionalmente, se realizó una clasificación retrospectiva del riesgo de progresión en el seguimiento de todos los pacientes, de acuerdo con los criterios de Miyauchi para la vigilancia activa del MCPT ${ }^{17}$. Se realizó análisis bivariado para el desenlace del compromiso ganglionar y los posibles factores asociados de acuerdo con la literatura. Se consideró con compromiso ganglionar significativo a los pacientes que presentaran cualquiera de los siguientes hallazgos: metástasis mayor de $3 \mathrm{~cm}$, más de 5 ganglios comprometidos y extensión extranodal; características que condicionan cambios en la clasificación posterior de riesgo de recaída, según las guías ATA $2015^{16}$. Finalmente, para este desenlace de interés, se realizó un modelo de regresión logística multivariado que incluyó todas las variables significativas en el análisis bivariado o que tuviesen relevancia clínica. Se utilizaron intervalos de confianza del $95 \%$ y todos los análisis se realizaron con Stata 15,0 (StataCorp LP, College Station, TX).

\section{Resultados}

De 1513 pacientes operados por patología tiroidea entre enero de 2017 y julio de 2020 en nuestra institución, 384 pacientes $(25,3 \%)$ presentaron tumores papilares de hasta $10 \mathrm{~mm}$ de diámetro y 286 se manejaron con tiroidectomía total más vaciamiento central, siendo esta la población de estudio.

Con respecto a las características clínicas, 254 pacientes $(88,8 \%)$ eran mujeres y 219 pacientes
(76,5 \%) tenían 55 años o menos (tabla 1). En los estudios prequirúrgicos, 43 pacientes $(15,0 \%)$ tenían ganglios sospechosos y un paciente $(0,3 \%)$ tuvo sospecha de compromiso extratiroideo en la ecografía, y se reportó la presencia de múltiples nódulos en el 48,9\% de los pacientes. En ninguno

Tabla 1. Características clínico-patológicas de los pacientes con microcarcinoma papilar de tiroides $(n=286)$

\begin{tabular}{|c|c|c|c|c|}
\hline \multicolumn{3}{|c|}{ Parámetro } & \multicolumn{2}{|l|}{ Frecuencia (\%) } \\
\hline \multirow[t]{2}{*}{ Sexo } & \multicolumn{2}{|c|}{ Mujeres } & \multicolumn{2}{|l|}{$254(88,8 \%)$} \\
\hline & \multicolumn{2}{|c|}{ Hombres } & \multicolumn{2}{|l|}{$32(11,2 \%)$} \\
\hline \multirow[t]{2}{*}{ Edad } & \multicolumn{2}{|c|}{ Menores de 55 años } & \multicolumn{2}{|l|}{$219(76,5 \%)$} \\
\hline & \multicolumn{2}{|c|}{55 años o más } & \multicolumn{2}{|l|}{$67(23,4 \%)$} \\
\hline \multicolumn{5}{|c|}{ Hallazgos prequirúrgicos en ecografía* } \\
\hline \multicolumn{3}{|c|}{ Ganglios sospechosos } & \multicolumn{2}{|l|}{$43(15,0 \%)$} \\
\hline \multicolumn{3}{|c|}{ Extensión extratiroidea } & \multicolumn{2}{|l|}{$1(0,3 \%)$} \\
\hline \multicolumn{3}{|c|}{ Nódulos múltiples } & \multicolumn{2}{|l|}{$140(48,9 \%)$} \\
\hline \multicolumn{5}{|c|}{ Hallazgos patológicos posquirúrgicos } \\
\hline \multicolumn{5}{|c|}{ Tumor primario $(\mathrm{T})$} \\
\hline \multicolumn{3}{|l|}{ T1a } & \multicolumn{2}{|l|}{$280(97,9 \%)$} \\
\hline \multicolumn{3}{|l|}{ T3b } & \multicolumn{2}{|l|}{$2(0,7 \%)$} \\
\hline \multicolumn{3}{|l|}{$\mathrm{T} 4 \mathrm{a}$} & \multicolumn{2}{|l|}{$4(1,4 \%)$} \\
\hline \multicolumn{5}{|c|}{ Ganglios linfáticos (N) } \\
\hline \multicolumn{3}{|l|}{$\mathrm{Nx}$} & $5(1,7 \%)$ & \\
\hline No & & & $140(48,9 \%)$ & \\
\hline N1 & & & $141(49,3 \%)$ & \\
\hline TNM e & tadio & & $<55$ años & $\geq 55$ años \\
\hline Estadi & & & $219(76.5 \%)$ & $47(16,4 \%)$ \\
\hline Estadi & & & $0(0 \%)$ & $19(6,6 \%)$ \\
\hline Estadi & & & NA & $1(0.3 \%)$ \\
\hline Tamañ & tumora & $\leq 0.5 \mathrm{~mm}$ & $49(17,1 \%)$ & \\
\hline & & $>0.5 \mathrm{~mm}$ & $237(82,9 \%)$ & \\
\hline Bilater & lidad & Sí & $57(19,9 \%)$ & \\
\hline & & No & $229(80,1 \%)$ & \\
\hline Multifo & alidad & Sí & $109(38,1 \%)$ & \\
\hline & & No & $177(61,9 \%)$ & \\
\hline Variant & histológ & ica papilar & & \\
\hline Clásicc & & & $212(74,1 \%)$ & \\
\hline Folicul & & & $62(21,7 \%)$ & \\
\hline Folicul & infiltrant & & $38(13,3 \%)$ & \\
\hline Folicul & encapsi & lado & $10(3,5 \%)$ & \\
\hline Célula & & & $5(1,7 \%)$ & \\
\hline Otros & & & $8(2,8 \%)$ & \\
\hline
\end{tabular}

*Predictores de alto riesgo para progresión de la enfermedad: Ganglios sospechosos en ecografía, extensión extratiroidea en la ecografía, variante histológica de alto grado en citológico 
de los casos se informó una variable histológica de alto grado en la punción con aguja fina.

De acuerdo con el informe de patología, se encontró que 280 pacientes $(97,9 \%)$ presentaron tumores de clasificación pT1a, 2 pacientes $(0,7 \%)$ fueron clasificados como pT3b y 4 pacientes $(1,4 \%)$ como pT 4 a. Todos los pacientes de menos de 55 años se clasificaron en estadio I de la enfermedad. Entre los pacientes de 55 o más años, el 70,2 \% se clasificaron en estadio I, el $28,3 \%$, en estadio II y el $1,5 \%$, en estadio III. El 17,1\% de los pacientes presentaron nódulos de hasta $5 \mathrm{~mm}$ de diametro y el 82,9 \% nódulos mayores de $5 \mathrm{~mm}$. El 38,1 \% tuvieron multifocalidad y el 19,9 \% tenían compromiso de ambos lóbulos. La variante histológica predominante fue la clásica, en 212 pacientes $(74,1 \%)$, seguida por la folicular, en 62 pacientes $(21,7 \%)$ y la folicular infiltrante, en 38 (13,3\%). Presentaron compromiso ganglionar en la patología, 140 (48,9\%) pacientes, y de estos, 47 pacientes $(33,6 \%)$ tenían más de 5 ganglios comprometidos o extensión extranodal. En ningún paciente se reportó metástasis mayor que $3 \mathrm{~cm}$ (tabla 2).

En cuanto a las complicaciones, 21 pacientes $(7,3 \%)$ presentaron disfonía postoperatoria y un paciente requirió traqueostomía. En el seguimiento a un año, se identificó hipoparatiroidismo definitivo de $0,8 \%$ de los pacientes y se reportó recaída de la enfermedad en 11 pacientes $(3,8 \%)$.

En el análisis de regresión logística, tanto bivariado como multivariado, se identificó que, para la edad menor de 55 años, el hallazgo de ganglios sospechosos en ecografía y nódulos mayores de 5 $\mathrm{mm}$, se asoció con la presencia tanto de compromiso ganglionar general, como de compromiso ganglionar significativo (tablas 3 y 4). Este riesgo se incrementó proporcionalmente, de acuerdo con el tamaño del tumor, por cada milímetro por encima de los $5 \mathrm{~mm}\left(\mathrm{OR}=1,22 ; \mathrm{IC}_{95 \%} 1,03-1,44 ; \mathrm{p}=0,019\right)$.

\section{Discusión}

A diferencia de numerosas patologías malignas en las cuales se prefiere intervenir en estadios tempranos, la tendencia en el MCPT es el manejo expectante a través de la estrategia de vigilancia activa, como fue propuesto por Miyauchi desde 1993, quien encontró que el $8 \%$ de los pacientes presentaron aumento del tamaño mayor que 3 mm y solo 3,8 \% tuvieron progresión ganglionar en más de 10 años de seguimiento ${ }^{21}$.

Tabla 2. Compromiso ganglionar en pacientes con microcarcinoma papilar de tiroides

\begin{tabular}{|c|c|c|c|}
\hline olac & esgo para prog & nto y con & 286) \\
\hline Compartimientos & $\begin{array}{c}\text { No } \\
\text { sin compromiso ganglionar }\end{array}$ & $\begin{array}{r}N \\
\text { compromiso gan }\end{array}$ & lionar presente \\
\hline $\begin{array}{l}\text { centrales evaluad } \\
\mathrm{n}=286 \mathrm{r}\end{array}$ & & Compromiso ganglionar general & Compromiso significativo * \\
\hline & Total: $146\left(51 \% ; \mathrm{IC}_{95 \%}: 45,1-56,9\right)$ & Total: $140\left(48,9 \% ; I_{95 \%}: 43,0-54,8\right)$ & $47 / 140\left(33,5 \% ; I_{95 \%}: 25,9-42,1\right)$ \\
\hline $\begin{array}{l}\text { Bajo Riesgo } \\
n=243\end{array}$ & 132 (54,3\%; IC $95 \%: 47,8-60,6)$ & 111 (45,7\%; IC $95 \%: 39,3-52,1)$ & $28 / 111$ (25,2 \%; IC $95 \%: 17,6-34,5)$ \\
\hline $\begin{array}{l}\text { Alto Riesgo } \\
n=43\end{array}$ & $14(32,6 \%$ IC $95 \%: 19,5-48,6)$ & $29\left(67,4 \% ; \mathrm{CC}_{95 \%}: 51,3-80,4\right)$ & $19 / 29$ (65,5 \%; IC $95 \%: 45,6-81,4)$ \\
\hline
\end{tabular}

Compromiso significativo ganglionar*

- Grupos de Riesgo de progresión en seguimiento

Total: $47(100 \%)$

$\begin{array}{ll}\text { Bajo Riesgo }(n=28) & \text { Alto Riesgo }(n=19) \\ 59,5 \%\left(I C_{95 \%}: 44,3-73,2\right) & 40,5 \%\left(I_{95 \%}: 26,7-55,6\right)\end{array}$

$\gamma$ : Pacientes llevados a vaciamiento ganglionar del compartimiento central.

*: Metástasis $>3 \mathrm{~cm},>5$ ganglios comprometidos, compromiso extranodal. 
Tabla 3. Análisis bivariado y multivariado de factores de riesgo relacionados con el compromiso ganglionar en pacientes con microcarcinoma papilar de tiroides

\begin{tabular}{lcccccc}
\hline \multirow{2}{*}{ Factor estudiado } & \multicolumn{3}{c}{ Análisis bivariado } & \multicolumn{3}{c}{ Análisis multivariado } \\
\cline { 2 - 7 } & OR & IC $_{95 \%}$ & $\mathbf{p}$ & OR & IC $_{95 \%}$ & $\mathbf{p}$ \\
\hline Edad < 55 años & 2,92 & $1,59-5,37$ & 0,001 & 3,14 & $1,63-6,05$ & 0,001 \\
\hline \multicolumn{1}{c}{$\begin{array}{l}\text { Mujer } \\
\text { Sexo }\end{array}$} & 1,26 & $0,60-2,65$ & 0,533 & & & \\
\hline $\begin{array}{l}\text { Ganglios sospechosos en } \\
\text { ecografía }\end{array}$ & 2,46 & $1,24-4,89$ & 0,010 & 2,75 & $1,32-5,74$ & 0,007 \\
Nódulos múltiples en ecografía & 0,74 & $0,46-1,20$ & 0,229 & 0,88 & $0,53-1,48$ & 0,651 \\
Tamaño del nódulo $>5$ mm & 3,19 & $1,61-6,33$ & 0,001 & 3,37 & $1,64-6,90$ & 0,001 \\
\hline
\end{tabular}

Tabla 4. Análisis bivariado y multivariado de factores de riesgo relacionados con el compromiso ganglionar significativo en el microcarcinoma papilar de tiroides

\begin{tabular}{lcccccc}
\hline \multirow{2}{*}{ Factor estudiado } & \multicolumn{3}{c}{ Análisis bivariado } & \multicolumn{3}{c}{ Análisis multivariado } \\
\cline { 2 - 7 } & OR & IC $_{95 \%}$ & $\mathbf{p}$ & OR & IC $_{95 \%}$ & $\mathbf{p}$ \\
\hline Edad < 55 años & 3,44 & $1,18-10,00$ & 0,023 & 3,84 & $1,24-11,85$ & 0,019 \\
\hline \multicolumn{1}{c}{ Mujer } & 0,66 & $0,27-0,12$ & 0,381 & & & \\
Sexo $\quad$ Hombre & & & & 1,53 & $0,52-4,43$ & 0,431 \\
\hline $\begin{array}{l}\text { Ganglios sospechosos en } \\
\text { ecografía }\end{array}$ & 6,07 & $2,96-12,47$ & 0,000 & 6,56 & $3,02-14,23$ & 0,000 \\
Nódulos múltiples en ecografía & 1,19 & $0,63-2,24$ & 0,591 & 1,60 & $0,78-3,27$ & 0,191 \\
Tamaño del nódulo > 5 mm & 3,49 & $1,03-11,75$ & 0,043 & 4,34 & $1,19-15,74$ & 0,025 \\
\hline
\end{tabular}

Esta conducta depende de la presencia o ausencia de características clínicas prequirúrgicas del paciente, como el hallazgo de variantes histológicas de alto grado, evidencia de compromiso ganglionar regional o a distancia, compromiso extratiroideo o crecimiento documentado en el seguimiento ${ }^{21}$. Lo anterior configura un grupo de alto riesgo de progresión, al que se le ofrece cirugía temprana, dejando a la población restante con MCPT en el grupo de bajo riesgo, donde es viable el seguimiento clínico e imagenológico. Sin embargo, la evidencia disponible en la literatura no es concluyente y deja la puerta abierta a discusiones.

$\mathrm{Si}$ bien la tendencia mundial es a disminuir el sobretratamiento en el cáncer de tiroides, los protocolos de seguimiento clínico son difíciles de aplicar en nuestro medio, en parte, debido a la escasa oferta de cirujanos de cabeza y cuello en nuestro país, especialmente fuera de las grandes ciudades, lo que limita frecuentemente una atención médica oportuna. Por otra parte, debido al temor de los pacientes y del cirujano a la pérdida de seguimiento por cuestiones administrativas de sus empresas prestadoras de salud (EPS) y otras condiciones sociales propias de la población de países en desarrollo, ante la sospecha de cáncer de tiroides, se opta frecuentemente por el manejo quirúrgico.

Actualmente, se discute el papel de la división por grupos de riesgo de progresión, en pacientes a quienes se les ha encontrado MCPT, como en el presente estudio, ya que la ausencia de características de alto riesgo no excluye el compromiso ganglionar significativo, lo cual afecta directamente el pronóstico del paciente ${ }^{22}$. Lo anterior sugiere 
que estos grupos podrían no ser aplicables a todas las poblaciones, y presenta la necesidad de estudios locales para determinar la mejor conducta en estos pacientes.

Al aplicar los criterios y clasificar por grupos, según lo propuesto por Miyauchi et al. ${ }^{9}$, en nuestra población de 286 pacientes, tan solo 43 pacientes hubiesen tenido indicación de manejo quirúrgico, por una clasificación prequirúrgica en alto riesgo, de los cuales $29(67,4 \%)$ tuvieron compromiso ganglionar general y $19(65,5 \%)$ tuvieron un compromiso ganglionar significativo, que aumentó su clasificación para riesgo de recaída según ATA 2015.

Por otra parte, de los 243 pacientes que, según lo propuesto por Miyauchi ${ }^{9}$, no tenían indicación de manejo quirúrgico por ser de bajo riesgo de progresión, 111 pacientes $\left(45,7 \%\right.$; IC $_{95 \%}$ : 39,3$52,1)$ tenían compromiso ganglionar, de los cuales 28 (25,2\%; IC $\left._{95 \%}: 17,6-34,5\right)$ correspondían a compromiso ganglionar significativo, lo que incrementa su clasificación de riesgo de recaída, con lo cual cambió la terapéutica adyuvante. Lo anterior refleja que, si solamente se hubiesen tenido en cuenta los hallazgos ecográficos y de citología para definir cuáles pacientes con MCPT se deben llevar a cirugía en nuestra población, no se hubiesen llevado a manejo quirúrgico una gran proporción de pacientes que desde el momento del diagnóstico tenían compromiso ganglionar importante.

De los 6 pacientes en quienes en la patología se documentó la extensión extratiroidea del MCPT, solo uno de ellos fue sospechado por imágenes preoperatorias. De igual manera, no se identificó una variante histológica de alto riesgo en la citología en ningún paciente, por lo que ninguna de estas dos variables se pudo tener en cuenta en el análisis de regresión logística del presente estudio. No hay mayores dudas en la literatura de que los pacientes con extensión extratiroidea del MCPT o en quienes de manera preoperatoria se documenta una variante histológica agresiva, deben ser manejados quirúrgicamente ${ }^{9,19}$.

Con base en lo anterior, al analizar los resultados posoperatorios, observamos que la correlación entre los grupos de riesgo de progresión de seguimiento y compromiso ganglionar no es buena, ya que los pacientes de bajo riesgo presentan compromiso ganglionar hasta en un $45,7 \%$, $\mathrm{y}$ al discriminar los datos por compromiso nodal significativo, el 59,5\% de los pacientes con compromiso significativo quedaron clasificados en el grupo de bajo riesgo, al que se le hubiese asignado vigilancia activa del nódulo.

Nuestros hallazgos permiten el debate en cuanto a la utilidad del manejo quirúrgico primario de los pacientes con MCPT asociado al vaciamiento central profiláctico (pCND, por sus siglas en inglés), ya que se disminuye la subestadificación que se presenta con la clasificación mediante características clínicas propuesta por Miyauchi et al. ${ }^{9}$, y las implicaciones que esto tiene para el pronóstico de los pacientes. Nuestros hallazgos van en concordancia con otros estudios que evalúan el pCND, en los que, en los pacientes con carcinoma diferenciado de tiroides (CDT), la realización del vaciamiento profiláctico lleva a cambio de la estadificación de los pacientes hasta en un $35 \%$, al pasar de N0 a N1a, especialmente en carcinomas menores de $1 \mathrm{~cm}$, entre los que está incluida nuestra población ${ }^{23}$. Además, se encontró que los pacientes llevados a pCND presentan una menor tasa de recurrencia local en el área central cervical $(1,0 \%$ vs $3,6 \%$; $<<0,01)$, lo cual evita reintervenciones con alta dificultad técnica y riesgo de complicaciones postoperatorias, como lesión del nervio laríngeo recurrente e hipocalcemia definitiva.

El grupo de cirugía de cabeza y cuello que desarrolló este estudio realiza sistemáticamente vaciamiento ganglionar central electivo a los pacientes con alta sospecha diagnóstica de cáncer de tiroides; sin embargo, este es otro punto de controversia, en relación con el mayor riesgo de complicaciones postoperatorias derivadas de una mayor extensión de la cirugía. En nuestra institución, para este grupo de pacientes, se detectó disfonía global transitoria o definitiva en el 7,3\% e hipoparatiroidismo definitivo en el $0,8 \%$, lo que se encuentra dentro de los estándares internacionales ${ }^{24}$. Esto permite concluir que, en esta población no se comprometió la seguridad del paciente al aumentar la extensión de la cirugía. 
Si bien se ha demostrado que el manejo expectante del MCPT es factible y seguro en nuestro medio, es necesario definir adecuadamente los criterios de elección para el protocolo de vigilancia activa y de esta forma evitar el retraso del tratamiento en pacientes que se presentan con enfermedad tumoral y compromiso nodal significativo. Es importante investigar en nuestra población, las características clínicas que orienten la decisión quirúrgica. Los hallazgos en el análisis bivariado y multivariado arrojaron datos estadísticamente significativos que relacionan la sospecha de compromiso ganglionar en ecografía preoperatoria, edad menor de 55 años y nódulos de más de 5 $\mathrm{mm}$, como factores independientes que aumentan el riesgo de compromiso ganglionar general y significativo.

Si bien se requieren más estudios, los hallazgos encontrados en nuestra población sugieren que los pacientes con las características mencionadas previamente podrían beneficiarse del manejo quirúrgico temprano, que adicionalmente puede incluir el pCND a criterio y experiencia del grupo tratante, el cual otorga beneficios en cuanto a la optimización de la estadificación y la reducción del riesgo de recaída local, siempre y cuando no se comprometa la seguridad del paciente.

\section{Limitaciones}

Este es un análisis ambispectivo de un solo centro, lo que disminuye la validez externa de los resultados. Adicionalmente, no se incluyeron todos los pacientes con diagnóstico de MCPT, dado que no todos fueron manejados con tiroidectomía total más vaciamiento central, lo que configura un sesgo de selección. Se realizó adecuado control del sesgo de información dado que la base de datos fue diseñada y dirigida al estudio, con el registro óptimo de las variables descritas.

\section{Conclusiones}

Los hallazgos de este estudio sugieren que el manejo quirúrgico temprano parece ser una opción adecuada en los pacientes con microcarcinoma papilar de tiroides que presentan sospecha de compromiso ganglionar en ecografía preoperatoria, edad menor de 55 años y nódulos de más de $5 \mathrm{~mm}$. Adicionalmente, en nuestra muestra, la clasificación en grupos de riesgo de progresión propuestos por Miyauchi ${ }^{9}$ no seleccionó adecuadamente los pacientes que se benefician de vigilancia activa, lo cual se demostró por el comportamiento del compromiso ganglionar significativo y su distribución en nuestros pacientes. Se requiere la implementación de estudios de carácter prospectivo para la aplicación y validación de los resultados obtenidos en el presente informe.

\section{Cumplimiento de normas éticas}

Consentimiento informado: Este estudio cumplió con las normas para la investigación en seres humanos, el protocolo y sus enmiendas fueron aprobadas por el Comité de Ética de la Investigación de la institución. Se obtuvo consentimiento informado de los pacientes. La confidencialidad y seguridad de la información fue garantizada usando la herramienta de RedCap y no se compartió información relacionada con la identificación de los pacientes en la preparación de este artículo.

Conflicto de interés: Ninguno declarado por los autores.

Fuente de financiación: Los recursos de financiación del proyecto provienen en su totalidad de aportes de los autores del proyecto de investigación.

\section{Contribuciones de los autores:}

- Concepción y diseño del estudio: Sergio Fabián ZuñigaPavia, David Mauricio Figueroa-Bohórquez, Pilar Carola Pinillos-Navarro.

- Adquisición de datos: Grupo de investigación del departamento de Cirugía general, Universidad Nacional de Colombia.

- Análisis e interpretación de datos: David Mauricio Figueroa-Bohórquez, Giancarlo Buitrago

- Redacción del manuscrito: David Mauricio FigueroaBohórquez, Pilar Carola Pinillos-Navarro, Jorge Alberto Martínez-Martínez, Daniela Casallas-Cristancho, Jenniffer Juliana León-Acero.

- Revisión crítica: Sergio Fabián Zuñiga-Pavia, Diego Alejandro Ardila-Torres, Giancarlo Buitrago.

\section{Referencias}

1. Davies L, Welch HG. Increasing incidence of thyroid cancer in the United States, 1973-2002. JAMA. 2006;295:2164-7. https://doi.org/10.1001/jama.295.18.2164 
2. Ross DS, Litofsky D, Ain KB, Bigos T, Brierley JD, Cooper DS, et al. Recurrence after treatment of micropapillary thyroid cancer. Thyroid. 2009;19:1043-8. https://doi.org/10.1089/thy.2008.0407

3. Davies L, Welch HG. Current thyroid cancer trends in the United States. JAMA Otolaryngol Head Neck Surg. 2014;140:317-22. https://doi.org/10.1001/jamaoto.2014.1

4. Ahn HS, Kim HJ, Welch HG. Korea's thyroid-cancer “epidemic"- screening and overdiagnosis. N Engl J Med. 2014;371:1765-7. https://doi.org/10.1056/NEJMp1409841

5. Mazzaferri E. Management of low-risk differentiated thyroid cancer. Endocr Pract. 2007;13:498-512. https://doi.org/10.4158/EP.13.5.498

6. Hay ID. Management of patients with low-risk papillary thyroid carcinoma. Endocr Pract. 2007;13:521-33. https://doi.org/10.4158/EP.13.5.521

7. Cadena E, Romero AE, Moreno A. Microcarcinoma papilar de tiroides (MCPT) metastásico a hueso. Revista Colombiana de Cancerología. 2011;15:98-103.

8. Ito Y, Miyauchi A, Inoue H, Fukushima M, Kihara M, Higashiyama T, et al. An observational trial for papillary thyroid microcarcinoma in Japanese patients. World J Surg. 2010;34:28-35. https://doi.org/10.1007/s00268-009-0303-0

9. Miyauchi A, Kudo T, Ito Y, Oda H, Sasai H, Higashiyama $\mathrm{T}$, et al. Estimation of the lifetime probability of disease progression of papillary microcarcinoma of the thyroid during active surveillance. Surgery. 2018;163:48-52. https://doi.org/10.1016/j.surg.2017.03.028

10. Romero-Rojas AE, Parra-Medina R, Chinchilla-Olaya SI, de los Reyes-Victoria A, Llamas-Olier A. Diferencias y controversias entre el reporte de patología y la interpretación clínica en patología tiroidea. I parte: Factores pronósticos. Revista Colombiana de Cancerología. 2017;21:160-5. https://doi.org/10.1016/j.rccan.2016.07.002

11. Sanabria A. Experience with active surveillance of thyroid low-risk carcinoma in a developing country. Thyroid. 2020;30:985-91. https://doi.org/10.1089/thy.2019.0522

12. Oh HS, Kwon H, Song E, Jeon MJ, Kim TY, Lee JH, et al. Tumor volume doubling time in active surveillance of papillary thyroid carcinoma. Thyroid. 2019;29:642-9. https://doi.org/10.1089/thy.2018.0609

13. Sugitani I, Fujimoto Y, Yamada K. Association between serum thyrotropin concentration and growth of asymptomatic papillary thyroid microcarcinoma. World J Surg. 2014;38:673-8. https://doi.org/10.1007/s00268-013-2335-8

14. Sánchez G, Gutiérrez C, Valenzuela A, Tovar JR. Carcinoma diferenciado de la glándula tiroidea: hallazgos en 16 años de manejo multidisciplinario. Rev Colomb Cir. 2014;29:102-109.
15. Zhang L, Wei WJ, Ji QH, Zhu YX, Wang ZY, Wang Y, et $a l$. Risk factors for neck nodal metastasis in papillary thyroid microcarcinoma: a study of 1066 patients. J Clin Endocrinol Metab. 2012;97:1250-7. https://doi.org/10.1210/jc.2011-1546

16. Wada N, Duh QY, Sugino K, Iwasaki H, Kameyama K, Mimura T, et al. Lymph node metastasis from 259 papillary thyroid microcarcinomas: frequency, pattern of occurrence and recurrence, and optimal strategy for neck dissection. Ann Surg. 2003;237:399-407. https://doi.org/10.1097/01.SLA.0000055273.58908.19

17. Kwak JY, Kim EK, Kim MJ, Son EJ, Chung WY, Park CS, et al. Papillary microcarcinoma of the thyroid: predicting factors of lateral neck node metastasis. Ann Surg Oncol. 2009;16:1348-55.

https://doi.org/10.1245/s10434-009-0384-x

18. Kim WY, Kim HY, Son GS, Bae JW, Lee JB. Clinicopathological, immunohistochemical factors and recurrence associated with extrathyroidal extension in papillary thyroid microcarcinoma. J Cancer Res Ther. 2014;10:505. https://doi.org/10.4103/0973-1482.131366

19. Haugen BR, Alexander EK, Bible KC, Doherty GM, Mandel SJ, Nikiforov YE, et al. 2015 American Thyroid Association Management Guidelines for Adult Patients with Thyroid Nodules and Differentiated Thyroid Cancer: The American Thyroid Association Guidelines Task Force on Thyroid Nodules and Differentiated Thyroid Cancer. Thyroid. 2016;261:1-133. https://doi.org/10.1089/thy.2015.0020

20. Ahn SH, Kim WS. The effect of prophylactic central neck dissection during hemithyroidectomy on locoregional recurrence in patients with papillary thyroid carcinoma: A meta-analysis. Clin Exp Otorhinolaryngol. 2020;13:194-202.

https://doi.org/10.21053/ceo.2019.01634

21. Miyauchi A. Clinical trials of active surveillance of papillary microcarcinoma of the thyroid. World J Surg. 2016;40:516-22. https://doi.org/10.1007/s00268-015-3392-y

22. Bradley NL, Wiseman SM. Papillary thyroid microcarcinoma: the significance of high risk features. BMC Cancer. 2017;17:142. https://doi.org/10.1186/s12885-017-3120-0

23. Liang J, Li Z, Fang F, Yu T, Li S. Is prophylactic central neck dissection necessary for $\mathrm{cN} 0$ differentiated thyroid cancer patients at initial treatment? A meta-analysis of the literature. Acta Otorhinolaryngol Ital. 2017;37:1-8. https://doi.org/10.14639/0392-100X-1195

24. Shan CX, Zhang W, Jiang DZ, Zheng XM, Liu S, Qiu M. Routine central neck dissection in differentiated thyroid carcinoma: A systematic review and meta-analysis. The Laryngoscope. 2012;122:797-804. https://doi.org/10.1002/lary.22162 